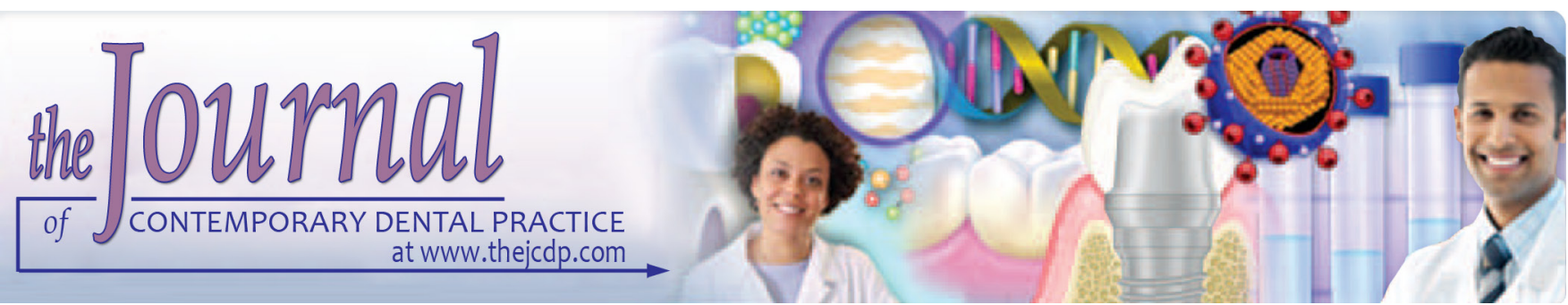

\title{
Bisphosphonate Induced Osteonecrosis of the Jaws: Our Clinical Experience at King Hussein Medical Center, Amman, Jordan
}

\author{
${ }^{1}$ Hytham Al-Rabadi, ${ }^{2}$ Luai K Daklalah, ${ }^{3}$ Mashoor Alwreikat, ${ }^{4}$ Mohammad Alqudah, ${ }^{5}$ Moath Momani, ${ }^{6}$ Hind F Nsour
}

\section{ABSTRACT}

Aim: To describe and analyze the clinical characteristics of bisphosphonate induced osteonecrosis of the jaws (BIONJ) patients diagnosed and treated at King Hussein Medical Center (KHMC), Amman, Jordan.

Patients and methods: A series of 12 patients with BIONJ was studied and analyzed retrospectively regarding age, gender, underlying disease, type, route and duration of bisphosphonate (BP) administered, site of osteonecrosis, initiating factor and treatment outcome were recorded. Follow-up period ranged from 6 months to 3 years.

Results: Patient's age ranged from 45 to 76 year old. Female to male ratio was $2: 1$. Most patients received IV BP as a part of the therapeutic protocol of their malignant disease; only two patients received oral BP. Mandible was more commonly involved compared to maxilla with a ratio of $5: 1$. In most patients the exposed necrotic bone was subsequent to an oral surgical intervention; However, in two cases the disease was initiated spontaneously. Complete wound healing was achieved in most of cases within a period ranged from 4 to 8 weeks. Two patients needed a second surgical intervention; However one patient was refractory to treatment and no wound healing achieved.

Conclusion: Bisphosphonate (BP) induced osteonecrosis of the jaws is a rare complication of bisphosphonates (BP) therapy.

\footnotetext{
1,3 Department of Oral and Maxillofacial Surgery, Royal Medical Services of Jordan Armed Forces, Amman, Jordan

${ }^{2}$ Department of Anesthesiology, Royal Medical Services of Jordan Armed Forces, Amman, Jordan

${ }^{4}$ Department of Periodontology, Royal Medical Services of Jordan Armed Forces, Amman, Jordan

${ }^{5}$ Department of Prosthodontics, Royal Medical Services of Jordan Armed Forces, Amman, Jordan

${ }^{6}$ Department of Pediatric Dentistry, Royal Medical Services of Jordan Armed Forces, Amman, Jordan
}

Corresponding Author: Hytham Al-Rabadi, Department of Oral and Maxillofacial Surgery, Royal Medical Services of Jordan Armed Forces, Amman, Jordan, Phone: 00962772114711, e-mail: hythamalrabadi@yahoo.com
Oral surgical intervention and female gender increase the risk of the disease. Early diagnosis and cessation of BP are essential to achieve success.

Clinical significance: It seems that some cases of BIONJ are refractory to the known treatment modalities. However, evaluation of treatment protocols may be needed in the future.

Keywords: Bisphosphonates, Mandible, Osteonecrosis, Retrospective study.

How to cite this article: Al-Rabadi H, Daklalah LK, Alwreikat M, Alqudah M, Momani M, Nsour HF. Bisphosphonate Induced Osteonecrosis of the Jaws: Our Clinical Experience at King Hussein Medical Center, Amman, Jordan. J Contemp Dent Pract 2018;19(11):1401-1404.

Source of support: Nil

Conflict of interest: None

\section{INTRODUCTION}

Antiresorptive medications such as BP became very popular in the last few decades due to their role in the therapeutic protocol of metastatic bony malignancies, such as multiple myeloma, breast, and prostate cancer, as well as for treatment of metabolic bony diseases, such as osteoporosis and Paget's disease. ${ }^{1}$

Bisphosphonates interrupt the mevalonate branch pathway, which is vital to the structural integrity and survival of osteoclasts, by inhibition of the farnesyl synthetase enzyme. ${ }^{2}$ It is used in medicine via two routes; intravenous (IV) and oral routes. Intravenous (IV) BP such as Amino bisphosphonates alendrenate (Aredia) and zoledronate (Zometa) are primarily used for management of malignant bony diseases. Oral BP, such as, amino bisphosphonates alendrenatee (fosamax) and residronate (Actonel) are used primarily for treatment of metabolic bony diseases. ${ }^{3}$

Despite the benefits of BP in medicine, BIONJ appeared as a rare side effect which have been claimed by many authors to be difficult to treat. ${ }^{4}$ 
The definition of BIONJ according to The American Association of Oral and Maxillofacial Surgeons is "Exposed bone in the maxilla or mandible that fails to heal within eight weeks in a patient receiving or who has received systemic bisphosphonate and who has not received local radiation therapy to the jaws". ${ }^{5}$

The aim of the present study is to describe and analyze the clinical characteristics of BIONJ patients diagnosed and treated at King Hussein Medical Center (KHMC), Amman-Jordan.

\section{PATIENTS AND METHODS}

The present retrospective study, which was approved by the ethical committee at KHMC in the Royal Medical Services, has included twelve patients who have presented to the department of oral and Maxillofacial surgery at KHMC between 2013 and 2016 with oral lesions following $\mathrm{BP}$ therapy. BIONJ was diagnosed according to the criteria established by the American Association of Oral and Maxillofacial Surgeons. ${ }^{5}$ No patient has received radiotherapy. Bone biopsy excluded the presence of bony metastasis. Age, gender, underlying disease, type, route and duration of $\mathrm{BP}$ administered, site of osteonecrosis, initiating factor and treatment outcome were recorded.

All patients were surgically treated by local surgical excision and debridement of necrotic bone after BP cessation, following coordination with the oncologist, for 6 months for those who were currently on BP therapy at the time when necrotic lesion appeared. Antibiotics were given according to microbiology culture. All patients were followed up for a period ranged from one to three years.

\section{RESULTS}

The presentation of BIONJ in all patients of this series was an area of exposed necrotic bone with variable bone involvement. Pain, swelling, recurrent bleeding, trismus,

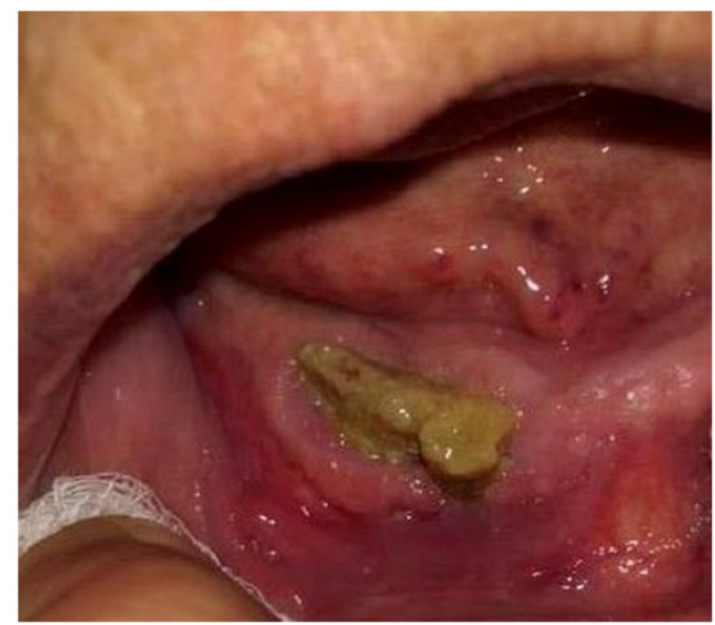

Fig. 1: Exposed nacrotic bone involving the mandible of BIONJ patient and teeth mobility were the most common associated symptoms (Fig. 1). the radiological findings were not specific with osteolytic lesion of variable extensions.

Patient's age ranged from 45 to 76 years with a mean age of 62 years. Female to male ratio was 2:1 (Table 1). Most patients received pamidronate and zoledronate IV BP as a part of the therapeutic protocol of their malignant disease (as multiple myeloma, breast and prostate cancer). Only two patients in our study received oral BP for treatment of osteoporosis. The time duration between starting BP therapy and the onset of oral symptoms was 34 months for IV BP and 38 months for oral BP.Mandible was more commonly involved compared to maxilla with a ratio of 5:1 (Table 2).

In most patients the exposed necrotic bone was subsequent to non-healing extraction socket, seven cases (Fig. 2), or following dental implant placement, two cases,and chronic denture trauma, one case. However in two cases the exposed necrotic bone appeared spontaneously with no history of any oral surgical intervention or trauma (Table 2).

Complete wound healing was achieved within a period ranged from 4 to 8 weeks for the two patients who were on oral $\mathrm{BP}$ and for seven patients of the ten who were on IV BP. Two patients needed another surgical intervention with re-excision with a wider safety margin before we achieved a complete wound healing, however one patient was refractory to treatment and no wound healing was achieved with the development of new necrotic bony lesion; this patient died during the follow-up period because of her malignant disease.

\section{DISCUSSION}

Bisphosphonates were administered for the first time to living animals in 1966; bone mass was increased. In 1990 the FDA approved alendronate, the oral BP,

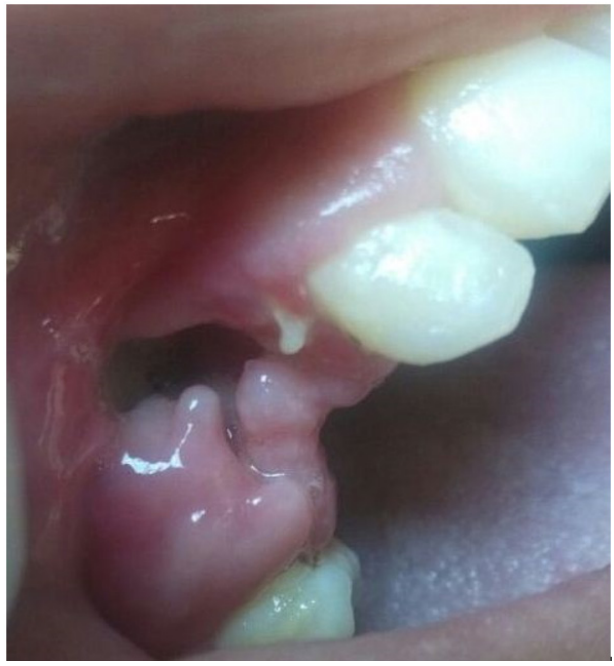

Fig. 2: BIONJ involving maxilla following dental extraction 
Bisphosphonate Induced Osteonecrosis of the Jaws (BIONJ)

\begin{tabular}{|c|c|c|c|}
\hline Case no. & Age & Gender & Primary disease \\
\hline 1 & 64 & $\mathrm{~F}$ & Breast cancer \\
\hline 2 & 55 & $\mathrm{~F}$ & Osteoporosis \\
\hline 3 & 76 & M & Prostate cancer \\
\hline 4 & 61 & $\mathrm{~F}$ & Breast cancer \\
\hline 5 & 77 & M & MM \\
\hline 6 & 45 & $\mathrm{~F}$ & MM \\
\hline 7 & 49 & $\mathrm{~F}$ & Breast cancer \\
\hline 8 & 55 & $\mathrm{~F}$ & Osteoporosis \\
\hline 9 & 70 & $\mathrm{M}$ & Prostate cancer \\
\hline 10 & 60 & $\mathrm{~F}$ & Breast cancer \\
\hline 11 & 59 & $\mathrm{M}$ & MM \\
\hline 12 & 75 & $\mathrm{~F}$ & Breast cancer \\
\hline
\end{tabular}

for the treatment of osteoporosis. Other oral BP, such as ibandronate and residronate, were approved. After that in 1991, pamidronate was approved as the first IV bisphosphonate, for the treatment of bone malignancies. ${ }^{6}$ The first report of osteonecrosis of the jaws related to the use of BP was by Marx in 2003. ${ }^{6}$ Hundreds of publications that relate osteonecrosis in the jaws to the use of IV or oral $\mathrm{BP}^{7}$ were published later.

Bisphosphonate induced osteonecrosis of the jaws (BIONJ) seems to be a rare complication of BP therapy; only twelve cases were diagnosed in our referral center during the period of three years.According to the American Association of Oral and Maxillofacial Surgeons the risk rate of developing BIONJ is about $0.5 \%$ after dental surgical procedures in patients who were received oral $\mathrm{BP}$, however, the risk ranges from 1.6 to $14.8 \%$ in patients who have received $\mathrm{BP}$ intravenously. ${ }^{5} \mathrm{BP}$ has an extremely rapid uptake into the skeleton; About $50 \%$ of the administered $\mathrm{BP}$ is absorbed within 30 minutes, and shows a high uptake affinity for areas of rapid bone turnover. ${ }^{8}$ Black and colleagues reported that the alveolar process demonstrates a tenfold increase in bone turnover compared to other parts of the skeleton; this may be the reason that BIONJ is diagnosed only in the jaws with no reported cases in other parts of skeleton. ${ }^{9}$

In the present series, $83 \%$ of BIONJ patients received IV BP and only $17 \%$ received oral $\mathrm{BP}$ with a shorter lag period between the initiation of BP therapy and osteonecrosis development with IV BP; the mean interval between initiation of BP therapy and the initiation of BIONJ was 34.6 months (34 months for IV $\mathrm{BP}, 38$ months for oral $\mathrm{BP}$ ). This is similar to reports by KOS et al. who found that most BIONJ patients have received IV BP with a small percentage of patients has received oral $\mathrm{BP} .{ }^{10} \mathrm{In}$ another series of patients with BIONJ reported by Jacobsen et al. the mean interval between initiation of BP therapy and initiation of lesions was 42 months. ${ }^{11}$

Table 2: BP route and duration, site of lesion, initiating factor and response to treatment

\begin{tabular}{clllll}
\hline $\begin{array}{c}\text { Case } \\
\text { no. }\end{array}$ & BP & $\begin{array}{l}\text { Duration } \\
\text { (months) }\end{array}$ & Site & Initiating factor & $\begin{array}{l}\text { Response } \\
\text { to } \\
\text { treatment }\end{array}$ \\
\hline 1 & IV & 37 & Mandible & Dental extraction & Yes \\
2 & Oral & 40 & Mandible & Dental implant & Yes \\
3 & IV & 34 & Maxilla & Dental extraction & Yes \\
4 & IV & 30 & Mandible & Dental extraction & Yes \\
5 & IV & 38 & Mandible & Spontaneous & Yes \\
6 & IV & 29 & Mandible & Denture trauma & Yes \\
7 & IV & 32 & Maxilla & Dental implant & Yes \\
8 & Oral & 36 & Mandible & Dental extraction & Yes \\
9 & IV & 33 & Mandible & Dental extraction & Yes \\
10 & IV & 36 & Mandible & Dental extraction & No \\
11 & IV & 35 & Mandible & Dental extraction & Yes \\
12 & IV & 36 & Mandible & Spontaneous & Yes \\
\hline
\end{tabular}

A higher incidence of BIONJ in mandible compared to maxilla was found in this series of BIONJ patients; this could be related to the higher vascularity of maxilla. This result was also documented by many authors. ${ }^{3,6,12}$ Li-Wan Lee, in his series of 40 BRONJ patients,founded that Twenty-seven (67.5\%) bone exposures occurred in the mandible only and $13(32.5 \%)$ in the maxilla only, he related this to the rich blood supply of the maxilla compared to mandible that make it more resistant to bone necrosis. ${ }^{3}$ However, Dimitrakopoulos et al. in his series found a higher incidence in maxilla compared to mandible. $^{2}$

In most cases in this study osteonecrosis developed following dento-alveolar surgery or trauma. Only two cases had developed the disease without any triggering factor. Similar findings were reported by Kos et al. ${ }^{10}$ in his series of 34 BIONJ patients, where only three patients lack an identifiable triggering factor.It is believed that patients who develop BIONJ spontaneously are more likely to have recurrences compared to patients who develop BIONJ after a surgical procedure. ${ }^{1}$ The only refractory case in the present series has developed the disease spontaneously without any triggering factor.

There were two times more females affected by the disease compared to males. It seems that gender can play a role in the susceptibility to BIONJ; which might be related to specific metabolic factors or to the fact that metabolic and malignant diseases treated by BP are more common in females. ${ }^{10,13} \mathrm{Li}$-Wan Lee, in his study group, consisted of $40 \mathrm{BRONJ}$ patients, reported a higher percentage of cases among females compared to males (65\% and $35 \%$ respectively). ${ }^{3}$

All patients in this series, presented with stage II disease (exposed necrotic bone with pain or signs of infection or both). The two patients with oral BP had complete remission of BIONJ lesions. Nine of our 10 patients who received IV BP also responded to 
treatment, two of them needed a second surgery, and one patient was refractory to treatment that was currently on BP at time of BIONJ diagnosis. Lopez-Cedrun et al. ${ }^{14}$ suggested in his series, of nine BIONJ patients that patients taking oral BP respond better to treatment than those taking IV BP.

It seems that the management of BIONJ is quite challenging. However, discontinuity of BP therapy, if possible, combined with surgical debridement to achieve clear and bleeding margins together with long- term antibiotic therapy administration is the treatment of choice. ${ }^{13,15}$ In our series the only patient who was refractory to treatment was the only one who was currently on BP at time of BIONJ diagnosis. Li-Wan Lee suggested that for BRONJ lesions, surgical excision and curettage can obtain a better and faster results bone healing than conservative treatment. ${ }^{3}$ Guilherme in his review article reviewed in details the possible treatment approaches for BIONJ reported in the literature in the last few years, In his data comparison he found that most reported studies in the literature include antibiotic therapy is more efficient for the management of BIONJ lesions when used in conjunction with bone surgery or debridement and hyperbaric oxygen therapy (HBO), which can induce collagen synthesis, angiogenesis and epithelization as a result of the increase in tissue oxygen tension. ${ }^{1}$ In the present study $\mathrm{HBO}$ was not used as a treatment modality because this facility was not available in our region.

The main limitation of the present study was the small number of our series; this was related to the minimal number of cases due to the relatively recent application of BP in medical therapy in our country and to the rarity of the disease which didn't allow us to collect more cases in our three years study period. We also didn't report the dose and the duration of BP therapy, however, many authors did not observe any relationship between the dose and the duration of BP and the development of osteonecrosis. $^{10}$

\section{CONCLUSION}

Bisphosphonate induced osteonecrosis of the jaws (BIONJ) is a rare complication of BP therapy. Oral surgical intervention and female gender increase the risk of the disease. Early diagnosis and cessation of BP are essential to achieve successful management.

\section{CLINICAL SIGNIFICANCE}

It seems that some cases of BIONJ are refractory to the known treatment modalities; management of BIONJ is quite challenging. Further evaluation of treatment protocols may be needed in the future.

\section{REFERENCES}

1. Ribeiroa GH, Chruna ES, Dutraa KL, Danielb FI, Grandob LJ. Osteonecrosis of the jaws: a review and update inetiology and treatment. Braz J Otorhinolaryngol. 2017 Jun 24

2. Dimitrakopoulos I, Magopoulos C, Karakasis D. Bisphosphonate-induced avascular osteonecrosis of the jaws: a clinical report of 11 cases. Int. J. Oral Maxillofac. Surg. 2006;35:588-593

3. Lee LW, Hsiao SH, Chen LK. Clinical treatment outcomes for 40 patients with bisphosphonates-related osteonecrosis of the jaws. Journal of the Formosan Medical Association 2014;113(3):166-172.

4. Kuijpers SCC, van Merkesteyn JPR: Bisphosphonate-related osteonecrosis of the jaws: Cohort study of surgical treatment results in seventy-four stage II/III patients. Journal of Cranio-Maxillofacial Surgery 2016.

5. American Association Oral Maxillofacial Surgeons. Position Paper on bisphosphonate- related osteonecrosis of the jaws. J Oral MaxillofacSurg 2007;65(3):369-376

6. Marx RE: Pamidronate (Aredia) and zoledronate (Zometa) induced avascular necrosis of the jaws: a growing epidemic. J Oral MaxillofacSurg 2003;61:1115-1117.

7. Sawatari Y, Marx RE. Bisphosphonates and bisphosphonate induced osteonecrosis.Oral MaxillofacSurgClin North Am. 2007 Nov;19(4):487-498.

8. Cariolatto FA, Carelli J, de Campos Moreira T, Pietrobon R, Rodrigues C, Bonilauri Ferreira AP. Recommendations for the prevention of bisphosphonate-related osteonecrosis of the jaw: a systematic review, The Journal of Evidence-Based Dental Practice (2018), doi: 10.1016/ j.jebdp.2017.11.002.

9. Black DM, Schwartz AV. Effects of continuing or stopping alendronate after 5 years of treatment: the Fracture Intervention Trial Long-term Extension (FLEX): a randomized trial.JAMA. 2006 Dec 27;296(24):2927-2938.

10. Kos M, Kuebler JF, Luczak K, EngelkeW. Bisphosphonaterelated osteonecrosis of the jaws: A review of 34 cases and evaluation of risk. Journal of Cranio-Maxillo-Facial Surgery 2010;38:255e-259.

11. Jacobsen C, Metzler P, Rossle M, Obwegeser J, Zemann W, Gratz KW. Osteopathology induced by bisphosphonates and dental implants: clinical observations. Clin Oral Investig 2013;7:167-175.

12. Marx RE, Sawatari Y, Fortin M, Broumand V. Bisphosphonate-induced exposed bone (osteonecrosis/osteopetrosis) of the jaws: risk factors, recognition, prevention, and treatment.J Oral Maxillofac Surg. 2005 Nov;63(11):1567-1575.

13. Antonio Chi-Kit Tong, George Kam-Hop Li, Wing-Yan Au. Bisphosphonate-induced Osteonecrosis of the Jaws:Report of 4 Patients. Asian J Oral Maxillofac Surg. 2009;21:59-63.

14. López-Cedrún JL,Sanromán JF, GarcíaA, Penarrocha M, Feijoo JF, Limeres J, DizP. Oral bisphosphonate-related osteonecrosis of the jaws in dental implant patients: a case series. British Journal of Oral and Maxillofacial Surgery 51 2013;874-87.

15. Pichardo SEC, Kuijpers SCC, van Merkesteyn JPR, Bisphosphonate-related osteonecrosis of the jaws: Cohort study of surgical treatment results in seventy-four stage II/III patients. Journal of Cranio-Maxillofacial Surgery 2016;44(9):1216-1220. 